\title{
"Volem Acollir": Humanitarismo y Posiciones de Sujeto en la Articulación de la Solidaridad en Cataluña
}

\author{
Álvaro Ramírez-March ${ }^{1}$ \\ ${ }^{1}$ Doctorando del programa Persona y Sociedad en el Mundo Contemporáneo y Investigador predoctoral \\ FPU (FPU17/01007) de la Universitat Autònoma de Barcelona. Barcelona, España. \\ E-mail: rezmarch@gmail.com; ORCID: https:/ / orcid.org/0000-0001-7029-8197 \\ Marisela Montenegro \\ 2Profesora titular del Departamento de Psicología Social de la Universitat Autònoma de Barcelona. \\ Barcelona, España. \\ E-mail: marisela.montenegro@uab.cat. ORCID: https:/ / orcid.org/0000-0001-7382-9256
}

\section{INTRODUCCIÓN}

En el contexto de un aumento de los movimientos migratorios de personas que huyen de conflictos políticos y otras situaciones de vulnerabilidad, así como del endurecimiento y mutación de las tecnologías fronterizas en la Unión Europea (UE) (Amoore, 2006; Vaughan-Williams, 2015), las nociones de frontera y asilo político se encuentran en el centro de una gran atención pública y académica (Suárez-Navaz, 2015; Sambaraju y McVittie, 2017).

Desde el otoño de 2015, las imágenes de los naufragios en el Mediterráneo y de las injustas situaciones que viven las personas en tránsito en las fronteras de la llamada "ruta de los Balcanes" se han vuelto familiares en los medios de comunicación del continente europeo (Georgiou y Zaborowsky, 2017). La situación, marcada por la vulnerabilización a la que se han visto expuestas estas personas, ha sido calificada por algunos autores como la "crisis humanitaria más grande desde la segunda guerra mundial" (Naïr, 2016:11). Ante esta coyuntura, las movilizaciones sociales en solidaridad con las personas migrantes y demandantes de asilo han tenido una considerable importancia en países que han recibido un gran número de personas. En lugares como Alemania (Langa, 2015), Italia (Grazioli y Caciagli, 2018) o Grecia (Kandylis, 2017), las iniciativas solidarias han sido clave para garanti- 
zar los Derechos Humanos ante la inabilidad o el fallo de los estados para hacerlo, siendo en ocasiones estas también el germen de nuevas formas de socialidad y organización (Papadopoulos y Tsianos, 2013).

En el caso del Estado español, la llegada de personas ha tenido una magnitud menor (CCAR, 2016), aunque las respuestas solidarias ante la situación se han hecho presentes desde diferentes niveles de la sociedad civil. Por ejemplo, a través del tránsito de personas voluntarias a lugares como Grecia; o bien a través de la organización de plataformas ciudadanas y grandes manifestaciones para presionar a las instituciones a desarrollar respuestas efectivas frente a la situación. En Cataluña, los sentimientos solidarios y críticos quedaron recogidos por iniciativas como "Stop Mare Mortum", durante finales de 2015 y 2016 y, centrándonos en el caso que hila este artículo, con la campaña mediática "Casa Nostra, Casa Vostra" (Nuestra Casa, Vuestra Casa), durante los meses de noviembre de 2016 a febrero de 2017.

Comenzada por un grupo de voluntarios que habían visitado los campos de refugiados cercanos a Thessaloniki (Grecia), esta iniciativa buscó generar un espacio para la articulación de distintos agentes sociales en favor de la acogida de demandantes de asilo. Después de una intensa actividad que incluyó, entre otras acciones, la retransmisión de pequeños documentales en prime time en la televisión pública catalana, la campaña concluía el 18 de febrero de 2017 con una gran manifestación bajo el lema "Volem Acollir" (Queremos Acoger).

En este artículo, exploramos la respuesta solidaria a la llamada "crisis de los refugiados" en Cataluña. Para ello, partimos de una primera reflexión sobre el título de la campaña para desgranar el contexto en el que esta tiene lugar. Así, si examinamos más detenidamente la expresión "mi casa es tu casa", podemos ver cómo esta da por hecho, en primer lugar, que existen las casas que son "de alguien" y, en segundo lugar, que estas casas pueden ser ofrecidas por un "nosotros" a un "otros". Estas dos figuras, la de la persona "que da" y la que "recibe", son ejemplos de dos posiciones que se encuentran implícitas en el discurso de la solidaridad construido dentro de la campaña. Tomando este ejemplo, indagamos en la manera en que estas dos posiciones han sido escenificadas en la generación de un movimiento solidario, quedando asociadas y atravesadas por distintos significados. 


\section{HERRAMIENTAS TEÓRICAS: POSICIONES DE SUJETO EN LA ARTICULACIÓN DE LA SOLIDARIDAD}

Para desplegar nuestro planteamiento, nos basamos en los desarrollos de Ernesto Laclau y Chantal Mouffe (2001; Laclau, 1993, 2004) sobre la naturaleza y la relación entre los ámbitos de lo social y lo político. Como Michel Foucault, Laclau y Mouffe plantean que el poder se encuentra disperso en lo social. En su pensamiento, estos autores hacen uso de la noción analítica de discurso, aunque existen, sin embargo, algunas diferencias en la manera en que llegan a ella. Para Foucault, que reflexiona sobre la historia, la preocupación principal es indagar sobre las relaciones entre determinados modos de saber y el poder, para así desentrañar los mecanismos por los que los seres humanos son convertidos en sujetos (Foucault, 2001: 241-259). Por su parte, Laclau y Mouffe se basan en una reinterpretación del concepto gramsciano de hegemonía, con el que proponen analizar los procesos de gobierno y organización política.

Las ideas de Gramsci cuestionan algunos aspectos clave de la teoría marxista, como la preponderancia de las estructuras económicas sobre otros dominios de lo social. Pero lo que es más importante, la concepción gramsciana de la hegemonía planteó que una clase social alcanza el poder (se convierte en hegemónica) no por imposición o dominación, sino a través de la representación de los intereses de las clases subalternas. De este modo, los procesos de formación de conciencia política - o "voluntad colectiva" en palabras de Gramsci - cobran un importante interés. La obra de Laclau y Mouffe se construye sobre esta idea, criticando, sin embargo, lo que entienden por "reductos esencialistas" en la teoría de Gramsci, para el cual el plano donde se constituyen los sujetos hegemónicos es "necesariamente, el plano de las clases fundamentales" (Laclau y Mouffe, 2001:181).

Por contraste, el análisis de los autores busca dar respuesta a un momento en el que la complejidad y diversidad de las luchas sociales excede la capacidad analítica del concepto de "clase". Así, entienden más bien que los modos de identificación que constituyen las distintas categorías sociales no pueden darse de forma independiente de la relación hegemónica que los constituye, que es siempre situada en un contexto sociohistórico. Las relaciones hegemónicas (lo social) son, por el contrario, entendidas como un campo multidimensional de fuerzas antagónicas marcado por relaciones "contingentes". En otras 
palabras, no existen identidades ni relaciones predeterminadas, sino que estas "son producto de una lucha hegemónica y de ello depende su sentido" (Laclau y Mouffe, 2001:208).

Las relaciones sociales (o los discursos, términos entre los cuales establecen una estrecha relación, utilizando uno u otro indistintamente) se definen como una totalidad que se estructura por resultado de una articulación de distintos significantes y posiciones de sujeto (Laclau y Mouffe, 2001:132). En una articulación discursiva, los distintos elementos de lo social adquieren su significado por una serie de tensiones entre relaciones de equivalencia y diferencia semántica dentro de una determinada estructura discursiva; mediante esta relación, los diferentes elementos modifican su identidad inicial. Estas tensiones no se dan únicamente entre equivalencia y diferencia, sino también entre los distintos elementos de la articulación, cuyos significados se sobredeterminan unos a otros. Es mediante esta sobredeterminación simbólica que se forman las relaciones de hegemonía: la operación mediante la cual una articulación discursiva adquiere su carácter "totalizante". Además, la hegemonía tiene lugar gracias a la presencia de lo que llaman "significantes vacíos", conceptos que mediante este juego de diferencias y equivalencias son "llenados" de significado y adquieren una preponderancia sobre los demás elementos de la estructura discursiva. De modo que este proceso en el que se generan las articulaciones hegemónicas es sólo posible por la producción social de los significantes, cuyo contenido es siempre contingente a un momento socio-histórico.

Siguiendo a Laclau, usamos el concepto de "posición de sujeto" como crítica a la idea del "sujeto" como origen de las relaciones sociales. Para este autor, un sujeto "es sólo parcialmente sujeto, [ya que] él también es un escenario de prácticas sedimentadas que organizan un marco normativo que opera como una limitación sobre el horizonte de opciones" (Laclau, 2004:90). En este sentido, las posiciones de sujeto "no están determinadas con anterioridad a la articulación discursiva que las constituye como tales, sino que es esta la que determina las condiciones de posibilidad discursivas" (Laclau y Mouffe, 2001:156), sobre las que las posiciones se establecen.

En nuestro caso, la perspectiva de Laclau y Mouffe nos es útil para preguntarnos por las condiciones sociales en las que se generan los significados y posiciones a partir de los cuales se construye la pro- 
blemática de la "crisis de los refugiados", así como los movimientos solidarios que le hacen frente, en la generación de una articulación discursiva determinada.

\section{METODOLOGÍA: CARTOGRAFIA DE LA SOLIDARIDAD}

Para desarrollar el análisis, partimos de una aproximación comprometida con la realidad que se estudia (Colectivo Situaciones, 2003; Casas-Cortés, Osterweil y Powell, 2013), haciendo uso de distintas metodologías puestas en práctica durante los meses de octubre de 2016 a diciembre de 2017. Estas se enmarcan en el proyecto "Cartografía de la Solidaridad", haciendo referencia la metáfora de la cartografía de Félix Guattari. Guattari describe la cartografía como un método capaz de "forjar nuevas coordenadas de lectura" (2000:32), o nuevas representaciones de la realidad que se estudia. Nuestro trabajo de campo se compone, en primer lugar, de observación participante en distintos espacios activistas de la ciudad durante los meses de la campaña, a partir de, entre otros espacios, reuniones de coordinación, manifestaciones y espacios públicos virtuales en defensa de derechos de las personas migrantes y en busca de asilo. En segundo lugar, de una serie de encuentros y talleres, enmarcados dentro de una experiencia de Investigación Acción-Participativa (IAP) (Montero, 2006), destinados a reflexionar sobre la llamada "crisis de los refugiados"; y en tercer lugar, de la construcción de diversas Producciones Narrativas (PN) (Balasch y Montenegro, 2003). Todas estas metodologías se entrelazan entre sí, de modo que fue a través de la observación participante que se establecieron las relaciones para desarrollar la IAP, y más tarde dentro de este marco se realizaron las distintas PN. En este artículo, basaremos la discusión en los significados que emergieron en las Producciones Narrativas realizadas.

La realización de una PN consiste en la producción de un texto híbrido a partir de, en un primer momento, una o varias sesiones donde investigadora y participantes hablan y discuten aspectos del fenómeno que se quiere estudiar, y en otro, la textualización de estas sesiones. Esto supone una revisión y reflexión de la sesión o sesiones en la que la conversación se traduce a un texto organizado y comunicable que refleja las posiciones y argumentos desarrollados a lo largo de estas. La "narrativización" es después remitida a la participante para que pueda modificar, corregir o expandir hasta que considere que esta refiere fielmente su punto de vista sobre el fenómeno. Se entiende, de esta manera, que la metodología busca fomentar la agencia de 
la participante, cuestionando la lógica clásica de investigación que divide sujeto/objeto de estudio y situando en ella la noción última de autoría. La metodología de las PN se basa en la perspectiva de los conocimientos situados de Donna Haraway (1991) y en su visión de los conocimientos como parciales y situados.

Para este trabajo se construyeron cuatro PN en colaboración con personas que forman parte de distintas iniciativas, todos ellas identificadas como significativas en la conformación de la respuesta solidaria con las personas migrantes y/o solicitantes de asilo en Cataluña. Las conversaciones que conformaron los productos textuales se compusieron, de una primera parte, en la que se habló de los significados asociados a la "crisis de los refugiados" y a la respuesta solidaria en Cataluña. En una segunda parte, se indagó sobre las relaciones entre las agentes sociales situadas en distintas posiciones, forjadas al calor de la campaña "Casa Nostra, Casa Vostra", que había finalizado hacía poco tiempo. Las cuatro personas participantes fueron:

1. Clara, de Comunitat Eko: iniciativa que surge de un grupo de personas voluntarias provenientes de Cataluña en el campo de refugiados de Idomeni, tras el cierre de la frontera de Grecia con Macedonia del Norte. Durante los primeros meses, el grupo trabajó para apoyar la autogestión en la vida diaria de un grupo de personas que esperaban a cruzar la frontera.

2. Núria, de Punt de Suport a les Persones Migrants, iniciativa que surge en la ciudad de Barcelona y que propone crear un espacio de apoyo mutuo entre activistas residentes y las personas demandantes de asilo recién llegadas o en tránsito.

3. Mustafa, del colectivo Espacio del Inmigrante, asentado en el barrio del Raval, Barcelona, creado a raíz de la exclusión del acceso a la sanidad gratuita para las personas irregularizadas en 2012. Desde aquel momento, el colectivo ha sido un espacio de empoderamiento del sujeto político migrante, así como de apoyo en cuestiones legales, sanitarias y psicosociales.

4. Pablo, técnico del Plan Ciudad Refugio, del Ayuntamiento de Barcelona, plan que surge en septiembre de 2015 para gestionar y coordinar los recursos de acogida de demandantes de asilo. 
En cuanto al análisis que se deriva de las $\mathrm{PN}$, a diferencia de otras metodologías, el tratamiento que estas reciben no es el de un producto puramente empírico, como material a analizar, ya que se entiende que esto supondría una posición de superioridad epistemológica de las investigadoras (Montenegro y Pujol, 2013). Al contrario, las narrativas resultantes se consideran comprensiones que sirven como puntos de partida para la reflexión teórica (Gandarias-Goikoetxea y García-Fernández, 2015). Siguiendo la concepción dialógica del lenguaje de Bajtin (1982 [1979]), la producción lingüística adquiere significado a partir de su aparición en "géneros del habla" propios de un contexto determinado. De este modo, las PN no son "muestras" del discurso público acerca de la "crisis de los refugiados", sino que entran en conversación con este y le responden haciendo uso de los géneros que circulan en ese momento. El análisis que presentamos, por tanto, busca mostrar cómo en las distintas conexiones de las investigadoras con las participantes, situadas todas en distintas posiciones de enunciación, se amplía la comprensión del fenómeno de la "crisis de los refugiados" y de "la respuesta solidaria" para, después, hilar estas distintas voces sobre la realidad en la construcción de una narrativa propia.

Partiendo de este marco teórico-metodológico, en este artículo analizamos en primer lugar el contexto que ha delimitado las condiciones de posibilidad discursivas sobre las que se han organizados las distintas iniciativas solidarias en Cataluña. A continuación, exploramos las posiciones de sujeto que han emergido en las relaciones solidarias como consecuencia de esta articulación discursiva. Por último, discutimos cómo este acercamiento permite un análisis crítico sobre las relaciones solidarias y de ayuda en este contexto.

\section{CRISIS, ¿CRISIS DE QUIÉN?}

Cuando hablamos de la crisis de los refugiados es fácil ponerle esta cursiva cuando escribimos, pero no tanto cuando hablamos. En este sentido, se habla de una crisis de los refugiados cuando la crisis no la generan los refugiados, la genera Europa en la atención de las personas que llegan a su territorio. Pablo, Ciutat Refugi (2017:6-7)

Como muestra el fragmento de Pablo, aunque la nomenclatura de la "crisis" es ampliamente criticada, esta se sigue utilizando profusamente. Para entender esto, es necesario considerar el papel de los medios de 
comunicación para la construcción de este fenómeno como un problema social, es decir, en su problematización. El punto cumbre de lo que ocurría en el Mediterráneo se construyó en el discurso público como una "crisis humanitaria" llegó en 2015 (Tsilimpounidi y Carastathis, 2017). En la primavera de este año, tuvo lugar una serie de naufragios en los que cientos de personas fallecieron en un intento de alcanzar las costas de la isla italiana de Lampedusa. En respuesta a la situación, la Comisión Europea declaró en una nota de prensa su intención de adoptar "medidas inmediatas para prevenir estas tragedias humanas y lidiar con las situaciones de emergencia" (EU Press Release, 27 de mayo de 2015). Haciéndose eco de esta narrativa, y alimentándola circularmente, los medios contribuyeron con su cobertura a la creación del marco de interpretación para la situación (Georgiou y Zaborowsky, 2017).

En el Estado español, la cobertura de la prensa vino a ser una continuidad de la de episodios similares, como los que siguieron a la desestabilización de Libia y el éxodo que esta produjo, contribuyendo a enmarcar a las personas que llegan a la UE en términos de una "amenaza" (como un problema "a solucionar"), por un lado, o bien como "víctimas" (con un problema a solucionar), en el caso de perspectivas humanitarias (Durán, 2017). Con todo, la llamada crisis queda definida como una preocupación por la llegada de un gran número de personas a la Unión Europea, así como por la dificultad de las distintas administraciones para gestionar su llegada y salvaguardar sus derechos.

El uso del término crisis (o emergencia) humanitaria sigue la tendencia desde la segunda mitad del siglo XX de asociar el término "humanitario" con los esfuerzos por mitigar las situaciones derivadas de las guerras y, en especial, de los efectos de grandes desplazamientos de personas. En los últimos años, sin embargo, el término "humanitario" ha sido usado principalmente para referirse a aquellas intervenciones realizadas ante el imperativo de la preservación y salvamento de vidas humanas. Según Craig Calhoun (2008), los términos "emergencia" o "crisis" humanitaria, con su llamada al imperativo moral de salvar vidas humanas, demandan una respuesta que se enuncia desde un lugar neutro, "más allá de la política". Esto, prosigue, funciona para naturalizar situaciones que en realidad son producto de la acción humana, presentando como eventos breves e impredecibles lo que en realidad son procesos y dinámicas más estructurales, sostenidas en el tiempo. De este modo, la "crisis de los refugiados" aparece como un evento excepcional, fruto de las guerras en ciertos países y el con- 
siguiente desplazamiento de personas. Al entender la situación como una "crisis", sin embargo, alejamos la mirada de lo que puede verse como un proceso continuado de movimientos migratorios, acompañado del endurecimiento y securitización de las tecnologías fronterizas en el Mediterráneo (Vaughan-Williams, 2015).

Iniciativas solidarias como Comunitat Eko critican los efectos de la crisis y se alejan de la concepción de esta como "excepcionalidad humanitaria".

Nosotros tenemos un punto bastante político, ya que no consideramos que sea una crisis humanitaria. Una crisis humanitaria es cuando hay un huracán y tú no puedes controlar y queda un territorio que está destruido, porque son cosas que pasan (...) Se trata de un conflicto político, un conflicto político desde Siria, Afganistán, Senegal (...) También lo es cuando los gobiernos europeos, en el momento cuando hay más muertos en el Mediterráneo, recortan presupuesto en salvamento militar y aumentan en construcción de fronteras y control policial. Lo que pasa en Siria es como la tercera guerra mundial pero externalizada. Es un conflicto político desde el momento en que se firma un tratado con Turquía, y por último con la gestión de los canales migratorios de entrada de esta Europa fortaleza, que es una apuesta política. (Clara, Comunitat Eko, 2017:5)

Convergiendo con esta idea, en su PN, Núria afirma que la situación puede ser reformulada teniendo en cuenta las relaciones de poder alrededor de la noción de frontera y las consecuencias que estas tienen en términos de clasificación, gestión y distribución de personas a partir de las distinciones que acarrean. De este modo,

La crisis de los refugiados es un proceso que hace muchos años que existe. Este proceso se basa en una política del cierre de fronteras, en la exclusión de la entrada a territorio europeo para las personas que no son deseables. Estas políticas se intensifican desde el 11 de septiembre de 2001, cuando se intensifican las restricciones en las fronteras a través de su externalización y la presencia muy clara de un discurso basado en la alteridad, con la asociación migrante-ilegal-terrorista. Esta última cuestión dificulta la entrada de ciertos grupos de personas en la UE, justificando su exclusión y bloqueo de las fronteras en base a una lógica de seguridad. No sólo se trata del cierre de las fronteras, sino de todo un sistema de deportaciones $\mathrm{y}$, a nivel institucional, de una política migratoria racista y excluyente. (Núria, Punt de Suport a les Persones Migrants, 2017:10) 
Lejos de ser excepcional, la situación que describe la crisis haría referencia a una serie de procesos de carácter político, económico y social de los cuales forma parte y cuyo análisis, por tanto, ha de ir en conjunto. Sin embargo, pese a todo, la narrativa de la "crisis" se impone en el discurso público.

En este sentido, Polly Pallister-Wilkins (2017) argumenta que la fuerza movilizadora del término "crisis" se encuentra contenida en la capacidad para enmarcar cuales son las respuestas éticamente posibles ante la situación en el Mediterráneo. Esto se puede observar en el énfasis gubernamental en las operaciones de búsqueda y rescate (Perkowsky, 2016; Tazzioli, 2016), pero también en la forma que son moldeadas las respuestas solidarias de la sociedad civil, que, de alguna manera, caen también dentro del campo de lo humanitario. En palabras de Miriam Ticktin, parece que "la única posición de sujeto disponible para aquellos que no están tratando de construir vallas o muros es la 'humanitaria'” (Ticktin, 2016:255, traducción propia). De este modo, aunque la nomenclatura de la crisis sea discutida, como llaman la atención estos fragmentos, resulta difícil matizar una situación que ha sido construida en estos términos de antemano.

En este artículo defendemos que los marcos de la "crisis" y la "aproximación humanitaria" han delimitado las condiciones de posibilidad discursivas que configuran las posiciones de sujeto en las movilizaciones solidarias que se han dado en Cataluña. A continuación, revisaremos cómo estos se reflejan en dos grandes posiciones que se corresponden con los dos lados de una relación de ayuda: en primer lugar, el refugiado, la víctima, no responsable de su situación y que además es entendida en términos "humanos"; y en segundo, las personas solidarias, aquellas que se sienten afectadas por las vivencias de ese "otro" y actúan. En una esfera distinta, pero también presentes, se encuentran los Estados, que no cumplen con su función de representación de estas personas - las ciudadanas comprometidas con "los refugiados" - y responden a otros intereses que llevan a un incumplimiento de sus funciones de auxilio.

\section{“CASA VOSTRA", ¿PERO A QUIÉN SE LA OFRECEMOS?}

La primera de las caras de la relación solidaria es la posición identificada como persona refugiada o "refugiado". Aunque esta denominación está lejos de ser la adecuada en todos los casos (la nomenclatura correcta para las personas con la intención de solicitar asilo político 
sería la de "solicitantes" o "demandantes" de asilo), ha sido esta la que ha trascendido al debate público. Más allá de los malentendidos que puede provocar la confusión, esta resulta también informativa del modo en que las categorías usadas para referirse a las personas que migran varían según el contexto sociohistórico en el que se utilizan.

Esta es la visión de Didier Fassin, para quien las categorías "migrante/ refugiado" no son estables, sino que están sometidas a transformaciones permanentes "mayormente causadas o al menos asociadas con cambios en la representación de los individuos involucrados y en la legitimidad de sus reivindicaciones" (Fassin, 2015:278). Esta "legitimidad" es a lo que el autor se refiere por "economía moral", y que define como la producción, circulación y apropiación de normas y obligaciones, valores y afectos relativos a un problema concreto en un tiempo y espacio específicos (Fassin, 2009). En su comentario sobre el contexto francés, Fassin (2015) narra el reciente cambio en las sociedades industrializadas occidentales en cuanto a la percepción de la migración "económica" y el asilo político. La primera pasó de ser percibida como "mano de obra necesaria" en las décadas posteriores a la segunda guerra mundial, a objeto de regulación y de rechazo con el cambio de la economía y la percepción de amenaza. En el caso del asilo político, el recelo hacia la migración habría provocado una actitud de sospecha que justificaría la concesión de cada vez menos solicitudes de asilo.

En el contexto que describimos, proponemos que las distintas cadenas de significación asociadas a estos términos habrían configurado una economía moral particular. Así, la identificación de las personas que cruzan el Mediterráneo con el significante "refugiado" en las circunstancias que son leídas como "una gran crisis humanitaria" habría vinculado la posición de sujeto "refugiado" con la categoría de "víctima", cuyos actos son responsivos a situaciones de violencia. Las víctimas, razón de ser del humanitarismo (Agier, 2010), son leídas en términos de "merecedoras de ayuda". Mientras tanto, aquellas otras personas que no encajan en esta categoría, aquellas etiquetadas como "migrantes económicas", son consideradas como no merecedoras (ver Holmes y Castaneda, 2016). En ese mismo sentido,

Antes de esta situación [la guerra de Siria] ya habían refugiados, que eran estas personas que cruzaban el Mediterráneo, solo que con el marco legal actual no se les podía conceder el estatuto de refugiado. Hablar de refugiados es escapar de la responsabilidad para no hablar de la causa y los 
problemas de los inmigrantes, algo que siempre ha existido. Creo que es un acto de hipocresía considerar que sólo estas personas tienen derecho a disfrutar del estatuto de refugiado, o el considerar que ellas son las únicas que sufren. En todo esto creo que han tenido mucha influencia los medios de comunicación. (Mustafá, Espacio del Inmigrante, 2017:5)

En el contexto de la campaña y de la formación de la respuesta solidaria en Cataluña, la distinción entre personas refugiadas "merecedoras" y migrantes "no merecedoras" se traduce en un "olvido" o infravisibillización de los problemas cotidianos de las personas migrantes ya viviendo en el territorio.

Este cambio en la economía moral, según Pablo, referiría a la relación que en los medios de comunicación se establece entre los "otros vulnerables" que vemos representados y las "sociedades de acogida" donde estas personas llegan.

Las fotos de las llegadas no son las barcazas de las personas negras, son de las personas que nos son más cercanas físicamente. Cuando se habla de los refugiados se hace únicamente de las personas que han huido de Siria y se encuentran en Grecia, pero no miramos a la frontera sur [del Estado español]. No pensamos en refugiados cuando vemos las imágenes de las personas que vienen de Ceuta y Melilla. (Pablo, Ciutat Refugi, 2017:9-10)

De este modo, la prominencia del caso sirio y la generación de una "responsabilidad" con respecto a este estarían influidas por la facilidad para despertar empatía, siendo mediada esta por la percepción de "cercanía social y cultural". Esto, como prosigue el participante, se debe a que la imagen que se ha transmitido a Europa de Siria es la de un país "casi laico, donde se podía viajar y por tanto se podía tener referentes" (Ciutat Refugi, 2017:9). Esta operación moral no se repite en el caso de las personas que cruzan por la frontera sur española, enclave con el que el Estado español sí tiene una relación histórica (colonial). La posición "refugiado", por tanto, aparecería delimitada por un entramado de "raza" y clase. 


\title{
"CASA NOSTRA": DIFERENCIAS Y TENSIONES EN LA EXPRESIÓN DE LA SOLIDARIDAD
}

Por otro lado, la segunda de las posiciones de sujeto que emerge en esta situación es la posición "solidaria". Se trata de la otra cara de la relación de ayuda, en la que otra persona o grupo decide responder a la llamada del "otro vulnerable". La relación solidaria aparece como una cuestión entre estos dos elementos ("refugiada" y "solidaria"), ambos contrapuestos al papel de la UE y de otras instituciones, consideradas como "culpables" de la situación. Sin embargo, el significado último de la "solidaridad" aparece como un significante vacío en disputa, que en función de las cadenas de significación que operan en el contexto adquiere uno u otro significado, todos ellos atravesados de una forma u otra por la razón humanitaria. A continuación, exploramos distintas maneras en las que esto sucede.

\section{Filántropo-nacionalismo y razón (post) humanitaria}

\begin{abstract}
La gente sale entonces a la calle [durante la manifestación "Volem Acollir"] para mostrar que se encuentra en una posición ética lejana de las decisiones que toma la Unión Europea. Aunque sea una muestra de solidaridad, estas manifestaciones muestran que también ellos sufren lo que hacen sus políticos en materia de política exterior. Al salir a la calle, quieren manifestar que se encuentran en una posición ética lejana a las instituciones. (Mustafá, Espacio del Inmigrante, 2017:8)
\end{abstract}

Los movimientos solidarios se han caracterizado por un rechazo al papel que han jugado las instituciones en la gestión de la situación. En el caso de Cataluña, sin embargo, el análisis adquiere varias capas de complejidad. La primera tiene que ver con la división de las competencias en materia de la gestión de las migraciones y el asilo político en el Estado español entre las distintas administraciones públicas. La segunda, en estrecha relación, es el actual proceso independentista del territorio y la confrontación del gobierno catalán con el gobierno central, por un lado, y entre el gobierno de Cataluña con el ayuntamiento de la ciudad de Barcelona, por otro; todos con posiciones distintas con respecto a la independencia de Cataluña. En Barcelona, la cronología de los acontecimientos se desenvuelve poco después de un cambio de gobierno local que coloca al partido de izquierda "Barcelona en Comú", y a Ada Colau, una conocida activista por los derechos de la vivienda, como alcaldesa de la ciudad. 
En la segunda mitad de 2015, durante los primeros meses de este "gobierno del cambio", se realizan diversas declaraciones institucionales que se unen a una creciente sensibilización social por la situación vivida en el Mediterráneo. El Ayuntamiento busca participar en la acogida de demandantes de asilo, crea el "Pla Barcelona Ciutat Refugi" e impulsa la "Red de Ciudades Refugio", que aglutina a varias ciudades en el ámbito estatal con el objetivo de presionar al gobierno central (de signo político contrario, en 2016); este, por su parte, rechaza las iniciativas de la Ciudad de Barcelona, reiterando que la competencia del asilo reside en la administración central.

En este complejo contexto tiene lugar la manifestación "Volem acollir". En ella, las demandas a las instituciones para acoger a solicitantes de asilo se articularon con discursos críticos sobre la gestión realizada por el Estado español. Se reclamaban, así, las competencias en materia de asilo (según la legislación, competencia estatal), con la presencia de pancartas con lemas como "Catalunya vol acollir, Espanya tanca fronteres" ("Cataluña quiere acoger, España cierra fronteras"). Estos carteles, junto con otros repartidos por asociaciones de la sociedad civil cercanas a una posición independentista, planteaban una diferenciación, en términos de valores más progresistas, de una futura "república catalana" dispuesta a acoger a personas refugiadas, en contraposición a el Estado español, responsable del cierre de fronteras. Esta posición, sin embargo, fue confrontada también por otras pancartas que huían de esta diferenciación entre políticos locales y estatales, pero además, y más importante, fue criticada por algunas entidades presentes en la manifestación, que veían en esta referencialidad a la comunidad de acogida una forma de instrumentalización por parte del movimiento independentista del discurso de la solidaridad con las personas migrantes y en busca de asilo.

\footnotetext{
En estos meses, colectivos como Eko y el Punt de Suport nos han apoyado para que los colectivos que estamos dentro de este bloque [el llamado "bloque migrante"] seamos visibles como representantes de las demandas de los migrantes, que sea nuestra voz la que sea escuchada, presionando juntos para conseguir que se eliminara la pancarta de la ANC, con la que no estábamos de acuerdo, y que se hablara más bien de la causa del Sindicato Popular de Vendedores Ambulantes. Mustafá, Espacio del Inmigrante (2017: 11)
}

Esta autorreferencialidad de la manifestación entra en conversación con lo que Lilie Chouliaraki (2011) llama un momento post-humanitario en la comunicación de la solidaridad con los "otros vulnerables". Lo post-hu- 
manitario señala un cambio en la expresión de la solidaridad en la que esta se alejaría de las llamadas a la acción en base a una cierta "humanidad compartida" con el otro vulnerable, para centrarse más en el yo y en las propias motivaciones para la acción. De este modo, las expresiones de solidaridad durante la manifestación estarían cargadas de una doble autorreferencia positiva: por un lado, a través de la confrontación con la actuación de la UE y, por el otro, en el caso independentista, a través de la oposición a la pertenencia y a la actuación del Estado español.

\section{Tensionando las lógicas humanitarias: activistas vs. cooperantes}

Además de las movilizaciones de presión hacia las instituciones, como la campaña "Casa Nostra, Casa Vostra", la expresión de la solidaridad ha tenido un papel importante a través del tránsito de personas voluntarias a lugares como Grecia, donde una gran cantidad de personas esperaban a ser trasladadas a otros países de la UE, dentro de los llamados acuerdos de "relocalización por tasas".

Unos meses antes de que se realizaran las conversaciones que dieron lugar a las Producciones Narrativas, en julio de 2016, tuvo lugar el campamento activista No Border Camp en la ciudad griega de Salónica. Estos eventos tienen su origen en la red antirracista europea del mismo nombre nacida en 1999. De afinidades antiautoritarias y anarquistas, esta busca un cambio radical más allá de la reforma de las leyes migratorias o del asilo (Alldred, 2003; Gauditz, 2017), llamando a la abolición de los controles fronterizos, los centros de detención y otras medidas punitivas que criminalizan la movilidad y crean precariedad. Tras el verano de 2015, que se caracterizó por una gran actividad y conflictividad cercana frontera de Grecia con Macedonia, este evento supuso un punto de encuentro para activistas de distintos países trabajando la temática. Entre sus objetivos estaba el intercambiar conocimientos y generar nuevos vínculos que fortalecieran las luchas por los derechos de las personas migrantes y la libertad de movimiento a través de las fronteras europeas (No Border Thessaloniki, 2016).

En el siguiente fragmento, situado espacialmente en el contexto del campamento activista, Núria recuerda su experiencia junto con un grupo de activistas que se desplazaron hasta la entrada de un "campo" para solicitantes de asilo. Viajando a las afueras de la ciudad, pretendían hacer llegar a sus habitantes unos periódicos elaborados desde la organización del evento. En el texto se informaba en 
griego, inglés y árabe de asuntos como el sentido y programación del No Border Camp, la situación política en materia migratoria o los esfuerzos de otras personas refugiadas en situaciones similares a las suyas para cruzar las distintas fronteras hasta llegar al resto de Europa. Este material no llegó a ser distribuido, como se cuenta, por la oposición de un grupo de personas identificados por Núria como cooperantes, que llevaban un tiempo trabajando en el campo, quienes justificaban su posición sobre la base del bien de las propias personas que habitaban el lugar.

[Las personas residentes] habían estado haciendo recientemente huelgas de hambre para protestar por la situación que vivían en el campo y estos cooperantes pensaban que si se volvían a politizar y salían a la calle acabarían frustrándose, aumentando la tensión y empeorando su situación, ya de por sí deplorable. Por esto, preferían que estuvieran tranquilos y no se politizaran. Esta actitud paternalista, que se justificaba en el bien de las personas de las cuales habían de hacerse cargo, se puede ver también como una manera de reforzar las estructuras que queremos combatir. (Núria, Punt de Suport a Persones Migrants, 2017:35-36)

En la narración de Núria, la posición de estas personas cooperantes se define por su juicio para discernir sobre lo que es bueno para las personas que viven en el campo, mientras que estas otras son vistas como "personas de las que cuidar". Esta actitud es considerada por las cooperantes como una conducta solidaria éticamente deseable, justificando el control sobre la capacidad de decisión de otras personas sobre la base de su cuidado. La dualidad entre cuidado y control es propia de los esfuerzos humanitarios por mantener unas condiciones dignas para la vida. Al mismo tiempo, se encuentra presente en la vigilancia y el control de las fronteras europeas, haciendo borrosa la frontera entre las dos prácticas (Pallister-Wilkins, 2017).

La sospecha de Núria en la última frase del fragmento, por otro lado, introduce un cuestionamiento de las lógicas humanitarias que actúan sobre las relaciones solidarias. Nos referimos a la crítica del humanitarismo como forma de gobierno - o gubernamentalidad (Fassin, 2012). El humanitarismo como forma de gubernamentalidad está presente en lugares como estos campos para personas refugiadas, donde, en palabras de Agier (2010), la gestión humanitaria adquiere un sentido de gestión de aquellas personas consideradas "indeseables". También en las intervenciones de salvamento marítimo en el Mediterráneo lle- 
vadas a cabo por agentes institucionales y no institucionales (Tazzioli, 2016; Stierl, 2017). En este otro caso, paradójicamente, aunque estas operaciones consiguen salvar las vidas de las personas que cruzan el Mediterráneo, después acaban entregando a las personas rescatadas a las autoridades portuarias, pasando a formar parte del engranaje de control migratorio y de deportación. En ambos casos, las intervenciones humanitarias pueden ser vistas como parte de la gubernamentalidad de la movilidad, insertándose y cambiando las formas en las que operan las tecnologías fronterizas de la UE.

La posición encarnada por el "activista" se ve, entonces, como una "resistencia" a esta "captura" por parte del poder soberano. Esta posición rechaza las políticas de sus propios Estados y reflexiona sobre la gubernamentalidad inherente a la aproximación humanitaria. Sin embargo, se encuentra paradójicamente reproduciendo las mismas relaciones de poder, puesto que estas son posibles precisamente a partir de las posiciones de sujeto que emergen de una situación de la que forman parte.

Cuando hablamos de la situación en Grecia y en los campos, me vienen a la cabeza debates internos y compartidos que teníamos algunas personas que habíamos ido allá con la idea de hacer activismo político, pero que nos habíamos encontrado desarrollando tareas humanitarias, como repartir comida en los campos. Vernos en esta dinámica reforzaba las posiciones asimétricas incluso más, ya que las personas a quienes ayudábamos se metían más en la posición de víctima y nosotras teníamos que poner límites con la misma gente. Era una gran contradicción que no era fácil de resolver: activismo y ayuda humanitaria se mezclaban continuamente. (Núria, Punt de Suport a Persones Migrants, 2017:37-38)

El marco de la "emergencia" sigue condicionando los modos de relación en estos contextos de ayuda, donde lo apremiante de la situación y el valor de la vida humana hace que se actúe a pesar de estar en desacuerdo con la aproximación con la que trabajan gobiernos y grandes instituciones, como el Alto Comisionado de las Naciones Unidas para los Refugiados (ACNUR). También, como contaba Núria más arriba, a pesar del desacuerdo con su propia ideología. 


\title{
FRONTERAS, SOLIDARIDAD Y POSICIONES DE SUJETO. HACIA UNA CONCLUSIÓN
}

\author{
Benvinguts, passeu passeu \\ de les tristors, en farem fum \\ que casa meva és casa vostra \\ si es que hi ha... cases d'algú
}

La campaña "Casa Nostra, Casa Vostra" toma el título de la utópica canción "Qualsevol Nit Pot Sortir el Sol" (Cualquier Noche Puede Salir el Sol), compuesta por el cantautor catalán Jaume Sisa, icono de la contracultura de los años 1970. La letra de la canción narra una invitación a una reunión con distintos personajes infantiles, culminando en el estribillo, reproducido más arriba, que invita a la persona que escucha a disfrutar de la hospitalidad del otro y unirse a los demás. Esta parte acaba, sin embargo, con la frase "si és que hi ha cases d'algúu" (si es que hay casas de alguien). Aquí, formulado en tono irónico, Sisa introduce la cuestión que ya insinuamos en la introducción: ¿puede existir la hospitalidad, la solidaridad, las relaciones de ayuda, sin los dos polos que constituyen esta relación?

Guiados por esta pregunta, en este texto hemos explorado la emergencia de estas dos posiciones en el contexto concreto de la gestación de una respuesta solidaria a la "crisis de los refugiados" en Cataluña. En primer lugar, esta respuesta ha estado afectada por la conformación de un espacio discursivo en el que la lógica humanitaria y el marco de la "crisis" han contribuido a generar una narrativa pública desprovista de un análisis estructural de la situación. Sin embargo, el análisis de "la crisis" y la "rutina" de los procesos de gobernanza y control de fronteras, así como del papel de la respuesta solidaria, ha de ir en conjunto (Pallister-Wilkins, 2017). Sólo así se podrá desgranar el papel de las dinámicas de poder desiguales que quedan ocultas por el marco de la excepcionalidad implícita en la noción de "crisis". Este marco, planteamos, ha actuado en el contexto que hemos presentado, influyendo en la emergencia de las dos posiciones de sujeto que, con ayuda de las Producciones Narrativas, hemos explorado.

Por un lado, la posición "refugiado" aparece reflejada como la "víctima", la razón de ser del humanitarismo (Agier, 2010), de cuyo reconocimiento como tal depende el ser "merecedor" de la ayuda solidaria. La generación de una determinada economía moral del asilo (Fassin, 2009; 
2015) habría reglado la circulación de la solidaridad. De este modo, en el seno de las relaciones solidarias con personas demandantes de asilo, se ha generado una nueva jerarquización y discriminación hacia aquellas otras personas que caen fuera de la categoría - las llamadas "migrantes económicas" - consideradas como "no merecedoras" de la solidaridad.

Por otro, la posición "solidaria" comprende varias aproximaciones con respecto a lo que son consideradas acciones adecuadas frente a la situación de los "otros vulnerables". La solidaridad, expresada como un rechazo al papel de las instituciones, provoca en el caso de Cataluña y del movimiento independentista, una suerte de "filántropo-nacionalismo". Llamamos así a las muestras de celebración de la entidad política catalana que la presentan como tolerante y progresista y dispuesta a ayudar, en contraposición al papel del Estado español responsable de la "no acogida" de solicitantes de asilo. En su auto-reflexividad, este tipo de expresiones adquieren tintes de una ética post-humanitaria, "que conserva la solidaridad hacia los otros, aunque sospechosa del papel de la política, se gira hacia uno mismo como fuente de una ética del cuidado" (Chouliaraki, 2013:3, traducción propia).

Precisamente, la posición "solidaria" se ha caracterizado por sus tensiones con la razón humanitaria. Su presencia influye en determinados tipos de solidaridad, donde las lógicas del cuidado y el control se confunden en pos del valor absoluto que se le otorga a la vida humana. Esta manera de funcionar, también presente en la fortificación y defensa de las fronteras europeas, invita a considerar los dos fenómenos, "cuidado" - o bienvenida - y "control" como parte del mismo proceso de gubernamentalidad de la movilidad humana (Chouliaraki y Georgiou, 2017). Por otro lado, las posiciones críticas con la aproximación humanitaria, que hemos identificado como "solidarias-activistas", buscan situarse - tanto en la discusión como en sus acciones - en un plano que permita denunciar las relaciones de poder presentes. No obstante, se encuentra participando también de la lógica humanitaria. $\mathrm{Y}$ en ocasiones, dado lo apremiante de ciertos contextos, navegan entre la urgencia de la atención y la politización de la situación.

Nos gustaría ahora volver a Jaume Sisa para ponerle en conversación con Jacques Derrida. En su libro On Hospitality (Derrida y Dufourmantelle, 2000), Derrida describe la hospitalidad como una "aporía", es decir, algo basado en su imposibilidad de existencia en una dimensión absoluta. Su 
razonamiento es el siguiente: si invitáramos a una persona a nuestra casa haciendo honor a la expresión "mi casa es tu casa" y esta persona tomara nuestro hogar como su hogar, las posiciones de huésped y anfitrión en las que se basa esta relación dejarían de existir. Para Derrida, la hospitalidad se basa en la existencia de una frontera, un espacio que separa las dos posiciones - la de huésped y anfitrión - y que es constitutivo de la relación. Este espacio, planteamos nosotros, también está presente en otras relaciones, como las solidarias o de ayuda.

Recuperando las ideas de Laclau y Mouffe (2001), podríamos argumentar que la propia acción solidaria, como una articulación que emerge en un contexto concreto, define las posiciones que se han presentado en el análisis y refuerza la díada entre aquellas personas o grupos que la "proveen" y aquellos otros que la "reciben". Desde el marco teórico de estos autores, el significante vacío "solidaridad" habría quedado asociado a otros significantes como "humanidad", "víctima", "humanitarismo" o "progresismo catalán", alrededor de los cuales se han constituido las posiciones de sujeto que hemos explorado. Así, las posiciones identificadas como contrarias a las lógicas de discriminación participan, aún desde la crítica y la acción directa, de la lógica de frontera que contribuye a generar estas dos posiciones.

Aunque pareciera que en este momento de nuestro argumento hayamos estrechado las posibilidades para la acción política, está lejos de ser nuestra intención. Con esta Cartografía, hemos querido llamar la atención sobre algunos aspectos problemáticos presentes en la generación de la respuesta solidaria a la llamada crisis. Mientras que esta ha sido importante para generar alternativas capaces de garantizar los derechos de aquellas personas en tránsito, creemos que esta crítica nos ayudará a pensar en formas de organización solidaria que puedan ser más efectivas en contestar las distintas discriminaciones. Ya que, volviendo por última vez a la teoría de Laclau y Mouffe (2001), las articulaciones hegemónicas no son nunca estables, sino que están siempre sujetas a indeterminaciones y cambios constantes. O en palabras de Laclau,

(...) toda identidad es dislocada en la medida en que depende de un exterior que, a la vez que la niega, es su condición de posibilidad. Pero esto mismo significa que los efectos de la dislocación habrán de ser contradictorios. Si por un lado ellos amenazan las identidades, por el otro están en la base de la constitución de identidades nuevas (1993:55). 
Si las posiciones de sujeto se constituyen a partir de dislocaciones de significados, esas dislocaciones abren, entonces, la posibilidad de nuevas formas de articulación y subjetividades políticas. Así, si bien dentro de esta postura se entiende que el sujeto emerge por una decisión altamente influida por la estructuración de poder existente (la constitución de eso llamado "solidaridad" en "la crisis de los refugiados"), este instaura a su vez una nueva estructura que excluye la posibilidad de otras decisiones e identificaciones en el futuro. Desde este punto de vista, la puerta quedaría abierta para que estas posiciones tengan la oportunidad de generar "nuevas dislocaciones" que, en un futuro, construyan a su vez nuevas articulaciones hegemónicas que cuestionen y modifiquen las fronteras, las geopolíticas, pero también las que se sitúan entre las posiciones de sujeto analizadas, y que desplacen, como en el análisis de Derrida, los límites de las relaciones de ayuda, hospitalidad y solidaridad.

(Recibido el 2 de julio de 2018)

(Reenviado el 19 de enero de 2020)

(Aprobado para su publicación el 2 de febrero de 2020)

\section{NOTAS}

1. La publicación de la foto del niño kurdo Aylan Kurdi después de que su embarcación naufragara tratando de cruzar hacia la UE y su posterior repercusión mundial marca el inicio de una gran atención de los medios de comunicación en distintos países de la UE (Georgiou y Zaborowsky, 2017).

2. Plataforma ciudadana con el objetivo de cambiar las políticas europeas de extranjería y migratorias. Su trabajo se basa en la sensibilización e incidencia política. Nace en 2015, tras la muerte de unas 900 personas en un naufragio en la costa italiana, con el objetivo de acabar con esta situación. Ver: http:/ / www.stopmaremortum.org

3. Según las personas organizadoras, se trató de "la manifestación más grande de Europa en favor de la acogida de personas refugiadas y migrantes" (Casa Nostra, Casa Vostra, 2018).

4. Durante este texto utilizaremos como genérico la forma femenina entendiendo que esta refiere a los nombres omitidos "persona" o "personas".

5. Las personas participantes acordaron que su nombre se hiciera público junto con sus Producciones Narrativas.

6. Hacemos uso del término personas "irregularizadas" y no "irregulares" para subrayar el papel de las leyes y regulaciones migratorias en generar categorías y jerarquías de discriminación. 
7. Texto original en catalán: “Quan parlem de la crisi dels refugiats és facil posar-li aquesta cursiva mentre escribim, però no tant quan parlem. En aquest sentit, es parla d'una crisi dels refugiats quan la crisi no la generen els refugiats, la genera Europa en l'atenció de les persones que arriben al seu territori".

8. Texto original en catalán: “Nosaltres teníem un punt bastant polític ja que no considerem que allò era una crisi humanitària. Una ciris humanitària és quan hi ha un huracà i tu no pots controlar i queda un territori que està destruït, perquè són coses que passen... Allò és un conflicte polític, un conflicte polític des de Síria, Afganistan, el Senegal... també ho és quan els governs europeus, en el moment on hi ha més morts a la Mediterrània, retallen pressupost en salvament militar i l'augmenten en construcció de fronteres i en control policial. El que passa a Síria és com la 3a guerra mundial però externalitzada. És un conflicte polític des del moment en que es firma un tractat amb Turquia, i a darrera la gestió de les canals d'entrada migratòria a Europa amb aquesta construcció de l'Europa fortalesa, que és una aposta política".

9. Texto original en catalán: “La crisi dels refugiats és un procés que fa molts anys que existeix. Aquest procés es basa en la política del tancament de fronteres, en l'exclusió de l'entrada al territori europeu de les persones que no son desitjables. Aquestes polítiques s'intensifiquen des de 1'11 de setembre del 2001 quan s'intensifiquen les restriccions a les fronteres a partir de la seva externalització i la presència molt clara d'un discurs basat en l'alteritat, amb l'associació migrant-il legal-terrorista. Aquesta última qüestió dificulta l'entrada de certs grups de persones a la UE, justificant la seva exclusió i el bloqueig de les fronteres en base a una lògica de la seguretat. No només es tracta del tancament de les fronteres, sinó de tot un sistema de deportacions i, a nivell institucional, una política migratòria racista i excloent".

10 Texto original en catalán: "Les fotos de les arribades no son les barcasses de les persones negres, són de les persones que ens son més properes físicament. Quan es parla de refugiats es fa únicament de les persones que han fugit de Síria i es troben a Grècia, però no mirem a la frontera sud. No pensem en refugiats quan veiem les imatges de les persones que venen de Ceuta i Melilla".

11. La Asamblea Nacional Catalana (en catalán Assemblea Nacional Catalana) es una organización de ámbito catalán, registrada como asociación, que tiene por objetivo alcanzar la independencia política de Cataluña (Wikipedia).

12. Asociación formada por vendedores ambulantes africanos que reivindica la despenalización de la venta ambulante en el espacio público. Durante el tiempo del proyecto han trabajado para generar otros imaginarios del trabajo informal, así como para señalar la relación entre este y la "irregularización" a la que los vendedores se ven sometidos por la Ley de Extranjería.

13. Debido a la llamada normativa de Dublín, estas personas están obligadas a pedir asilo en el primer país de la UE al que llegan. Los reasentamientos, acordados por los distintos gobiernos de la UE en mayo y septiembre de 2015, estaban destinados a "aliviar" la carga de los países a los que estas personas habían llegado inicialmente, buscando un "reparto" entre los distintos países de la UE. El 27 de septiembre de 2017, fin del plazo de dos años para llevar a cabo las relocalizaciones, España sólo había recibido unas 1938 personas, un 11\% del total que su compromiso inicial (CEAR, 2017). 
14. La figura del "cooperante" hace aquí referencia a la idea de "cooperación internacional". En el contexto catalán y español, este campo se identifica con intervenciones transnacionales que pretenden hacer llegar ayuda humanitaria a comunidades donde se necesitan. Ver al respecto una perspectiva histórica y local en la Producción Narrativa de Clara, activista de Mujeres Palante (2018).

15. Texto original en catalán: “(Les persones residents al camp) recentment havien estat fent vagues de fam per protestar per la situació que vivien al camp i aquests cooperants pensaven que si es tornaven a polititzar i sortir al carrer acabarien frustrant-se, augmentant la tensió i empitjorant la seva situació, ja per si deplorable. Per això preferien que estiguessin tranquils i no es polititzessin. Aquesta actitud paternalista, que es justificava en el mateix bé de les persones de les quals s'havien fet càrrec, es pot veure també com una manera d'enfortir les estructures que volem combatre".

16. Texto original en catalán: “Quan parlem de la situació a Grècia i als camps, em venen al cap debats interns i compartits que teníem algunes persones que havíem anat allà amb la idea de fer activisme polític, però que ens trobàvem desenvolupant tasques humanitàries, com repartir menjar als camps. Veure'ns en aquesta dinàmica reforçava les posicions asimètriques fins i tot més, ja que les persones a les quals ajudàvem es ficaven més en la posició de víctima i nosaltres havíem de posar límits amb la mateixa gent. Era una gran contradicció que no era fàcil de resoldre: activisme i ajuda humanitària es barrejaven contínuament".

17. En castellano: Bienvenidos, pasad, pasad / de las tristezas, haremos humo / que mi casa es tu casa / si es que hay...casas de alguien. 


\section{REFERENCIAS}

AGIER, Michel. (2010), "Humanity as an identity and its political effects (a note on camps and humanitarian government)". Humanity: An International Journal of Human Rights, Humanitarianism, and Development, vol. 1, $\mathrm{n}^{\circ} 1$, pp. 29-45.

ALLDRED, Pam. (2003), No borders, no nations, no deportations. Feminist Review, vol. 73, pp. 152-157.

AMOORE, Louise. (2006), "Biometric borders: Governing mobilities in the war on terror". Political Geography, vol. 25, n³, pp. 336-351.

BALASCH, Marcel; MONTENEGRO, Marisela. (2003), “Una propuesta metodológica desde la epistemología de los conocimientos situados: Las producciones narrativas". Encuentros en Psicología Social, vol. 1, n 3, pp. 44-48.

BAJTÍN, Mijail. (1979), Estética de la creación verbal. México: Siglo XXI.

CALHOUN, Craig. (2008), "The imperative to reduce suffering: charity, progress and emergencies in the field of humanitarian action". In: M. Barnett; T. G. Weiss (eds.), Humanitarianism in question: politics, power, ethics. Ithaca and London: Cornell University Press.

CASA NOSTRA, CASA VOSTRA. (2017), Merecen un futuro mejor: Queremos Acoger I Casa Nostra, Casa Vostra. Disponible en http:/ /www.casanostracasavostra.com/es / quienes-somos/organizacion. Recuperado en $1^{\circ}$ de julio de 2018.

CASAS-CORTÉS, Maribel; OSTERWEIL, Michal; POWELL, Dana E. (2013), "Transformations in engaged ethnography: knowledge, networks, and social movements". En: J. S. Juris; A. Khasnabish (eds.), Insurgent encounters: transnational activism, ethnography $\mathcal{E}$ the political. Durham and London: Duke University Press.

CCAR, Comissió Catalana d'Ajuda al Refugiat. (2016), Informe 2016: Les persones refugiades en Europa i en Espanya. Resumen Ejecutiu. Comissió Catalana d'Ajuda al Refugiat. Recuperado a partir de http:/ / www.ccar.cat/publicacions-i-recursos/informes-cear / informe-2016/

CEAR. (2017), Informe 2017: Las personas refugiadas en España y Europa. Recuperado a partir de https:/ / www.cear.es/wp-content/uploads/2017/06/Informe-Anual-CEAR-2017.pdf

CHOULIARAKI, Lilie. (2011), “Improper distance': towards a critical account of solidarity as irony". International Journal of Cultural Studies, vol. 14, nº 4, pp. 363-381.

(2013), The ironic spectator: solidarity in the age of post-humanitarianism. Cambridge, Malden, MA: Polity Press.

; GEORGIOU, Myria. (2017), "Hospitability: the communicative architecture of humanitarian securitization at Europe's borders. Journal of Communication, vol. 67, $\mathrm{n}^{\circ} 2$, pp. 159-180.

CIUTAT REFUGI. (2017), Ciutat Refugi - Cartografia de la Solidaritat. Disponible en https:/ / archive.org/details/ciutatrefugicsol.

COLECTIVO SITUACIONES. (2003), Sobre el Militante Investigador. Disponible en http: / / eipcp.net/transversal/0406/colectivosituaciones/es. Recuperado 1 de julio de 2018. 
COMUNITAT EKO. (2017), Comunitat Eko - Cartografia de la Solidaritat. Disponible en: https:/ / archive.org/details/ekocsolidaritat/

DERRIDA, Jacques; DUFUORMANTELLE, Anne. (2000), Of Hospitality. Palo Alto, CA: Stanford University Press.

DURÁN, Rafael. (2015), “El problema de los otros. Encuadre de inmigrantes y/o desplazados en la Lampedusa de la primavera árabe". Revista de Estudios Políticos, vol. 0, n 169, pp. 299-331. https://doi.org/10.18042/cepc/rep.169.10

EUROPEAN COMISSION. (25 de mayo de 2015), Press release: La Comisión Europea avanza en la Agenda de Migración. Disponible en http://europa.eu/rapid/press-release_IP15-5039_es.htm. Recuperado en 2 de julio de 2018.

FASSIN, Didier. (2009), "Les économies morales revisitées". Annales. Histoire, Sciences Sociales, vol. $64, \mathrm{n}^{\circ}$ 6, pp. 1237-1266.

. (2012), Humanitarian reason: a moral history of the present. Berkeley, CA: University of California Press.

. (2015), “La economía moral del asilo. Reflexiones críticas sobre la 'crisis de los refugiados' de 2015 en Europa". Revista de Dialectología y Tradiciones Populares, vol. 70, $\mathrm{n}^{\circ} 2$, pp. $277-290$.

FOUCAULT, Michel. (2001), “El poder y el sujeto”. En H. L Rabinow; P. Dreyfus (eds.), Michel Foucault: más allá del estructuralismo y la hermenéutica. (pp. 241-259). Buenos Aires: Nueva Visión.

GANDARIAS-GOIKOETXEA, Itziar; GARCIA-FERNÁNDREZ, Nagore. (2015), "Producciones narrativas: una propuesta metodológica para la investigación feminista". En: I. Mendia Azkue et al. (eds.), Otras formas de (re) conocer. Reflexiones, herramientas y aplicaciones desde la investigación feminista (pp. 97-110). Donostia-San Sebastian: Hegoa.

GAUDITZ, Leslie. (2017), “The noborder movement: interpersonal struggle with political ideals". Social Inclusion, vol. 5, no 3, 49-57. https://doi.org/10.17645/si.v5i3.968.

GEORGIOU, Myria; ZABOROWSKY, Rafal. (2017), Council of Europe report. Media coverage of the "refugee crisis": a cross-European perspective. Council of Europe. Disponible en: https://rm.coe.int/1680706b00.

GRAZIOLI, Margherita; CACIAGLI, Carlotta. (2018), Resisting to the neoliberal urban fabric: housing rights movements and the re-appropriation of the 'right to the city' in Rome, Italy. VOLUNTAS: International Journal of Voluntary and Nonprofit Organizations. https: / / doi.org/10.1007/s11266-018-9977-y

GUATTARI, Felix. (2000), Cartografías esquizoanalíticas. Madrid: Manantial.

HARAWAY, Donna J. (1991), Simians, cyborgs, and women: the reinvention of nature. New York: Routledge.

HOLMES, Seth M.; CASTAÑEDA, Heide. (2016), “Representing the 'European refugee crisis' in Germany and beyond: deservingness and difference, life and death". American Ethnologist, vol. 43, no 1, pp. 12-24. 
KANDYLIS, George. (2017), "Urban scenes of citizenship: inventing the possibility of immigrants' citizenship in Athens". Citizenship Studies, vol. 21, no 4, pp. 468-482. https:/ / doi.org/10.1080/13621025.2017.1307606

LACLAU, Ernesto. (1993), Nuevas reflexiones sobre la revolución de nuestro tiempo. Buenos Aires: Nueva Visión.

. (2004), “Identidad y hegemonía: el rol de la universalidad en la constitución de lógicas políticas". En: J. Butler; E. Laclau; S. Zizek (eds.), Contingencia, hegemonía y universalidad. Diálogos contemporáneos en la izquierda. Buenos Aires: Fondo de Cultura Económica.

; MOUFFE, Chantal. (2001), Hegemonía y estrategia socialista. (2 ed.) Madrid: Siglo XXI.

LANGA, Napuli. (2015), "About the refugee movement in Kreuzberg/Berlin". Movements. Journal für kritische Migrations- und Grenzregimeforschung, vol. 1, no 2. Recuperado a partir de http://movements-journal.org/issues/02.kaempfe/08.langa--refugee-movement-kreuzberg-berlin.html

MONTERO, Maritza. (2006), Hacer para transformar. El método de la psicología comunitaria. Buenos Aires: Paidós.

MONTENEGRO, Marisela; PUJOL, Joan. (2013), “Producciones narrativas: una propuesta teórico-práctica para la investigación narrativa”. En: M. Rodigou; H. Paulín (eds.), Coloquios de investigación Socialex (pp. 15-42). Córdoba. Recuperado a partir de http:/ / generatech.org/sites/default/files/coloquioii.pdf

MUJERES PALANTE. (2018), Caminando juntas en el activismo. Disponible en: https:/ / archive. org/details/CaminandoJuntasEnElActivismoClaraMujeresPalante

NAÏR, Sami. (2016), Refugiados: frente a la catástrofe humanitaria, una solución real. Recuperado a partir de https:/ / dialnet.unirioja.es/servlet/libro?codigo=671085

NO BORDER THESSALONIKI. (2016), A collection of texts presented at Thessaloniki's No Border Camp. Recuperado a partir de http://noborder2016.espivblogs.net/files/2017/08/NoBorder2016_Papers.pdf

PALLISTER-WILKINS, Polly. (2017), “Humanitarian rescue/sovereign capture and the policing of possible responses to violent borders". Global Policy, vol. 8, pp. 19-24. https:// doi.org/10.1111/1758-5899.12401

PAPADOPOULOS, Dimitris; TSIANOS, Vassilis S. (2013), "After citizenship: autonomy of migration, organisational ontology and mobile commons". Citizenship Studies, vol. 17, $\mathrm{n}^{\circ} 2,178-196$.

PAPELES PARA TODOS Y TODAS. (2017), Papeles para todos y todas - cartografia de la solidaritat. Disponible en https: / / archive.org/details/papersxtothomcsol

PERKOWSKY, Nina. (2016), “Deaths, interventions, humanitarianism and human rights in the mediterranean 'migration crisis'”. Mediterranean Politics, vol. 21, nº 2, pp. 331-335.

PUNT DE SUPORT DE PERSONES MIGRANTS. (2017), Punt de suport de persones migrants - cartografia de la soliaritat. Disponible en http:/ /archive.org/details/puntdesuportcsol

SAMBARAJU, Rahul; MCVITTIE, Chris. (2017), “The European Union and the refugee 'crisis': inclusion, challenges, and responses". Journal of Community \& Applied Social Psychology, vol. 27, nº 2, pp. 99-104. 
STIERL, Maurice. (2017), "A fleet of mediterranean border humanitarians". Antipode, n/a-n/a. https://doi.org/10.1111/anti.12320

SUÁREZ-NAVAZ, Liliana. (2015), “Introducción. Etnografías y emergencias en el Mediterráneo: agujeros negros de nuestra modernidad". Disparidades. Revista de Antropología, vol. $70, \mathrm{n}^{\circ} 2$, pp. 265-276.

TAZZIOLI, Martina. (2016), “Border displacement: challenging the politics of rescue between Mare Nostrum and Triton". Migration Studies, vol. 4, no 1, pp. 1-19.

TICKTIN, Miriam. (2016), "Thinking beyond humanitarian borders". Social Research: An International Quarterly of the Social Sciences, $\mathrm{n}^{\circ}$ 2, pp. 255-271.

TSILIMPOUNIDI, Myrto; CARASTATHIS, Anna. (2017), “The 'refugee crisis' from Athens to Lesvos and Back: a dialogical account". Slovak Ethnology, $\mathrm{n}^{\circ} 65$.

VAUGHAN-WILLIAMS, Nick. (2015), Europe's border crisis: biopolitical security and beyond. Oxford, New York: Oxford University Press. 


\section{RESUMO}

"Volem Acollir": Humanitarismo e Posições de Sujeito na Articulação da Solidariedade em Catalunha

A chamada "crise dos refugiados" no Mar Mediterrâneo tem provocado uma grande mobilização social de solidariedade. Este artigo parte de Produções Narrativas realizadas com iniciativas solidárias que participaram em uma campanha cidadã em Catalunha para analisar: 1) as implicações de sua problematização em termos de "crises"; e 2) as posições de sujeito que emergem na articulação da resposta solidária. Exploramos as duas posições que se correspondem com as duas caras de uma relação de ajuda: por um lado, a do "refugiado", associada ao imaginário da "vítima"; e por outro, a "solidária", que dá uma resposta ao "outro vulnerável". Defendemos que ambas as posições, bem como o marco da "crise", são influenciadas por uma lógica humanitária. Concluímos que este marco é problemático por não abranger a complexidade da situação e gerar novas lógicas discriminatórias.

Palavras-chave: solidariedade; humanitarismo; posição de sujeito; Catalunha; crise dos refugiados

\section{ABSTRACT \\ "Volem Acollir": Humanitarism and Subject Positions in Manifestations of Sympathy in Catalonia}

The so-called "refugee crisis" of the Mediterranean has caused vast demonstrations of social sympathy. This article uses narrative productions from sympathy initiatives which took part of a citizen campaign in Catalonia as the basis for the analysis of: 1) the implications of problematizing it in terms of "crises"; and 2) the subject positions which emerge from manifestations of sympathetic response. We explore two positions which correspond to two faces of an aid relation: on the one side, "refugees", associated to the imaginary of "victims"; on the other, "the sympathetic", who provide a response to "vulnerable others". We propose that both positions, as well as the "crisis" benchmark, are influenced by a humanitarian logic. We conclude that such benchmark is problematic, since it does not comprehend the situation's complexity and it generates new logics of discrimination.

Keywords: sympathy; humanitarianism; subject positions; Catalonia; refugee crisis 


\section{RÉSUMÉ}

"Volem Acollir": Humanitarisme et Positions de Sujet dans l'Articulation de la Solidaritéen Catalogne

La soi-disant "crise des réfugiés" en mer Méditerranée a provoqué une grande mobilisation sociale de solidarité. Cet article part de productions narratives menées avec des initiatives de solidarité qui ont participé à une campagne citoyenne en Catalogne pour analyser: i) les implications de leur problématisation en termes de "crises"; et ii) les positions du sujet qui émergent dans l'articulation de la réponse solidaire. On explore les deux positions qui correspondent aux deux visages d'une relation d'aide: d'une part, celle du "réfugié", associée à l'imaginaire de la "victime"; et de l'autre, le "solidaire", qui répond aux "autres vulnérables". On soutient que les deux positions, ainsi que le cadre de "crise", sont influencés par une logique humanitaire. On conclut que ce jalon est problématique car il ne couvre pas la complexité de la situation et génère de nouvelles logiques discriminatoires.

Mots-clés: solidarité; humanitarisme; position du sujet; Catalogne; crise des réfugiés

\section{RESUMEN \\ "Volem Acollir": Humanitarismo y Posiciones de Sujeto en la Articulación de la Solidaridad en Cataluña}

La llamada "crisis de los refugiados" en el Mar Mediterráneo ha provocado una gran mobilización social de solidaridad. Este artículo parte de Producciones Narrativas realizadas con iniciativas solidarias que participaron en una campaña ciudadana en Cataluña para analizar 1) las implicaciones de su problematización en términos de "crisis"; y 2) las posiciones de sujeto que emergen en la articulación de la respuesta solidaria. Exploramos dos posiciones que se corresponden con las dos caras de una relación de ayuda: por un lado, la del "refugiado", asociada al imaginario de la "víctima" y, por el por otro, la "solidaria", aquella que da una respuesta al "otro vulnerable". Defendemos que ambas posiciones, así como el marco de la "crisis", son influidas por una lógica humanitaria. Concluimos que este marco resulta problemático al no abarcar la complejidad de la situación y generar nuevas lógicas discriminatorias.

Palabras clave: solidaridad; humanitarismo; posición de sujeto; Cataluña; crisis de los refugiados 